\title{
TEORÍAS DE LA TIERRA Y SISTEMAS GEOLÓGICOS: UN LARGO DEBATE EN LA HISTORIA DE LA GEOLOGÍA"
}

\author{
Francisco Pelayo \\ Dpto. Historia de la Ciencia - Centro de Estudios Históricos (CSIC)
}

\section{RESUMEN}

Durante la segunda mitad del siglo XVIII se emitieron las primeras teorías del orígen e historia de la Tierra. La interpretación que consideraba al Diluvio como el factor geológico decisivo en el modelado de la superficie terrestre fue la más debatida hasta el surgimiento de los sistemas geológicos catastrofista y actualista en el siglo XIX. En la actualidad, continua abierta la polémica entre geólogos e historiadores de la ciencia sobre métodos y sistemas en la Historia de la Geología.

\begin{abstract}
During the second half of the XVIIIth century, the first theories about the origin and history of the Earth were issued. The interpretation that considered Deluge as a decisive factor in the modelling of land surface was the most debated until the issue of the Catastrophism and Actualism geological systems in the XIX'th century. Norwadays, the polemics about methods and systems in the History of Geology is still open between geologists and historians of science.
\end{abstract}

\section{INTRODUCCIÓN}

La Geología es una disciplina científica que se ha caracterizado prácticamente desde sus orígenes por dar una gran importancia al aspecto histórico-crítico. Desde que en 1729 el naturalista suizo Louis Bourguet realizara un primer intento de clasificación de las distintas hipótesis emitidas sobre la teoría de la Tierra, que él remontaba a los clásicos, hasta las críticas geológicas de Georges Cuvier y Charles Lyell, del primer tercio del siglo XIX, vertidas respectivamente desde el catastrofismo y el uniformitarismo, la mayor parte de los filósofos y naturalistas que propusieron durante este período su propia interpretación de la geología histórica, iniciaban su discurso comentando, analizando y criticando las obras de aquellos de sus predecesores que

* Trabajo realizado en el marco del Proyecto de la DGCYT PB94-0060. 
habían descrito un sistema geológico con el que pretendían explicar el origen y el desarrollo histórico de la Tierra.

Esta tradicional metodología histórico-crítica, característica de las ciencias geológicas, ha continuado durante las últimas décadas, concretándose en una frecuente aparición de trabajos publicados, principalmente por historiadores de la ciencia y paleontólogos, en los que se ha discutido la validez metodológica del catastrofismo y del uniformismo, y que han abierto un debate de gran interés debido al nuevo marco catastrofista en el que se discute actualmente la interpretación del pasado de la Tierra.

\section{LAS IDEAS GEOLÓGICAS DE LOS AUTORES CLÁSICOS.}

Al plantearse en sus alusiones a los fósiles el problema de su procedencia, los filósofos griegos propusieron diferentes opiniones acerca de la historia de la Tierra. Jenófanes de Colofón (570-480 a. de C.), por ejemplo, llegó a suponer la existencia de inundaciones periódicas de las tierras, que causaban la muerte de los hombres al hundirse los continentes en el mar, apareciendo posteriormente una nueva generación de organismos. Según Kindermann ${ }^{1}$, esta explicación de Jenófanes estaba relacionada con una concepción catastrofista del mundo, según la cual, las fuerzas físicas habían operado en el pasado con mayor intensidad y energía que en el presente, lo que había provocado grandes cataclismos universales.

Distinta iba a ser la postura de Aristóteles (384-322 a. de C.), partidario de la eternidad del mundo. En su opinión, éste se desgastaba y reparaba en todo su conjunto, originándose cambios en la superficie terrestre entre los espacios ocupados por el mar y los continentes ${ }^{2}$. Los cambios tenían lugar cuando diluvios locales o grandes inundaciones causadas por lluvias torrenciales anegaban los continentes, al tiempo que el mar retrocedía de su antiguo lecho dejando regiones que quedaban en seco. De esta forma se mantenía el equilibrio entre tierra y agua. En una línea parecida fueron las concepciones actualistas de Estraton de Lampsaca (335-269 a. de C.) y Eratóstenes (276-196 a. de C.), quienes atribuyeron las conchas marinas halladas en tierra a su deposición durante el lento retroceso del mar. Estos autores fueron criticados por Estrabón (64 a. de C.- 24 d. de C.), quien, cercano a posturas catastrofistas, pensaba

\footnotetext{
1 Puede verse Kindermann, U. (1981), "Conchae marinae. Marine Fossilien in der Fachliteratur des frühen Mittelalters", Geologische Blätter für Nordost-Bayern, Erlangen, 31, pp. 516-517.

2 Véase ARISTOTELES, Les Météorologiques, Paris, Vrin, (I, 14), pp. 72-81. También puede consultarse sobre este tema ElLENBERGER, F. (1989), Historia de la Geología, M.E.C. y Labor, p. 23.
} 
que era necesario el concurso de causas de mayor energía, como terremotos, erupciones volcánicas, levantamientos del suelo submarino y hundimientos súbitos ${ }^{3}$.

Platón (428-347 a. de C.), por su parte, exponía en el Timeo que a intervalos regulares, aunque muy espaciados de tiempo, tenían lugar en la Tierra cataclismos que provocaban la muerte de los hombres. Atribuía las mayores de estas destrucciones al fuego y al agua, que en su texto sólo parecían afectar al exterminio de los seres vivos, sin que hiciera referencia a ningún tipo de alteración del relieve geológico causada por estas catástrofes cíclicas ${ }^{4}$. A pesar de esto último, se puede considerar que la concepción cosmogónica de los platónicos, al igual que la de los estoicos, estaban más cercanas de lo que posteriormente se denominaría catastrofismo geológico.

Sin duda, el fragmento literario que más influyó en las concepciones geohistóricas de los eruditos y naturalistas de la Edad Moderna fueron ocho versos que Ovidio (43 a. de C.-17 d. de C.), en Las Metamorfosis, libro XV, versos 262-269, puso en boca de Pitágoras (580-500 a. de C.), y que hacían referencia a los cambios geológicos acaecidos en el tiempo.

Dentro de la concepción catastrofista de los estoicos, partidarios de la existencias de conflagraciones universales cíclicas seguidas de un proceso de renovación, hay que destacar la exposición que hizo el filósofo estoico hispano-romano, Lucio Anneo Séneca (siglo I) sobre fenómenos geológicos y atmosféricos, recogida en sus Naturales Quaestiones. Al tratar el tema de las aguas terrestres, Séneca exponía cómo la Tierra sería inundada por un diluvio universal, que destruiría toda la vida existente, aunque posteriormente todos los organismos serían creados de nuevo ${ }^{5}$. Séneca pensaba que dicha "revolución" geológica no se haría sin provocar un violento desastre en el globo terrestre, y sostenía que el gran cataclismo se originaría por la concurrencia de una serie de causas como lluvias y crecidas de los ríos y los mares, que se saldrían de sus límites.

A partir del siglo IV, con el establecimiento del cristianismo como religión oficial del Estado, surgió en Europa Occidental un nuevo modo de acercarse a la naturaleza. En la concepción cristiana de la historia del mundo, el diluvio bíblico iba a desempeñar un papel aún más importante que el que la cosmogonía estoica le atribuía a la catástrofe diluvial, ya que todos los estudios sobre el pasado de la naturaleza tuvieron como punto de partida los relatos de la creación y del diluvio del Génesis ${ }^{6}$. 21.

3 GoHau, G. (1990), Les sciences de la Terre aux XVII' et XVIII' siècles, Paris, Albin Michel, p.

4 Platon, Timeo. Edición consultada de Buenos Aires, Aguilar, 1981, 4ª edición, pp. 81-84.

5 SENECA, L. Los ocho libros de las cuestiones naturales. Edición consultada Buenos Aires, Espasa Calpe, 1948, pp. 45-54.

6 Conviene recordar que el relato del diluvio bíblico no era original del pueblo hebreo sino que era una narración tomada de fuentes babilónicas anteriores, que describía un suceso que había tenido lugar en Mesopotamia. Sin embargo, al recogerse en el Génesis, se convirtió en Europa Occidental durante 


\section{FRANCISCO PELAYO}

\section{LAS "TEORÍAS DE LA TIERRA" Y EL ORIGEN DEL DILUVISMO EN EL SIGLO XVII.}

Se puede situar como mediados del siglo XVII la época en que se postularon las primeras propuestas que sirvieron de base a las posteriores "teorías" o sistemas de la Tierra. Partiendo de dos modelos, una explicación mecanicista del origen de la Tierra, emitida por Descartes, y una hipótesis diluvista de la historia del relieve terrestre, expuesta por el filólogo español J. A. González de Salas, que al no ser mutuamente excluyentes terminaron fundiéndose, se emitieron durante más de un siglo y medio diferentes "Teorías de la Tierra", comentadas y criticadas por sucesivos autores, quienes a su vez propusieron sus propios sistemas geológicos ${ }^{7}$.

\section{La teoría de la Tierra de Descartes.}

La hipótesis heliocentrista sugerida por Nicolás Copérnico (1473-1543) a mediados del siglo XVI, cuya defensa habría de costar tan cara a Galileo, fue un factor decisivo para que desde los años cuarenta del siglo XVII se discutiera en la comunidad científica europea el problema del origen y la inestabilidad histórica de la corteza terrestre. Este interés por el pasado de la Tierra surgió cuando ésta, creada por Dios con el único fin de servir como morada al hombre según la Biblia, perdió su anterior posición privilegiada en el centro del universo, para ocupar un lugar más modesto entre los planetas, ya que este cambio provocó un reconocimiento de la importancia de la Tierra en cuanto escenario en el que se había desarrollado la historia del género humano.

El primer autor del siglo XVII que centró sus especulaciones en los acontecimientos geológicos que habían tenido lugar en el pasado fue René Descartes (1596-1650). La hipótesis de Descartes acerca del origen de la Tierra como un antiguo sol apagado, planteada al margen del relato de la creación del Génesis, sirvió como punto de 
partida a las posteriores discusiones sobre la geología histórica ${ }^{8}$. Sin embargo, es preciso decir que Descartes fue muy cauto al redactar su explicación mecanicista del origen de la Tierra, que entraba en contradicción con el creacionismo del Génesis. Consciente de los problemas que había tenido Galileo con la ortodoxia católica, desde el comienzo de su obra se apresuró a dejar claro que su interpretación de la Tierra como un antiguo sol extinto sólo la planteaba como una hipótesis? ${ }^{9}$.

En la cuarta parte de su tratado Principia Philosophiae (Paris, 1644) ${ }^{10}$, Descartes expuso la que se considera la primera teoría de la Tierra ${ }^{11}$. Algunas de las cuestiones

8 Sobre la obra geológica de Descartes puede verse DaubréE, A. (1880), "Descartes, l'un des créateurs de la cosmologie et de la géologie", Journal des Savants, marzo, pp. 165-175 y abril, pp. 208221.

9 $\quad$ Ibidem, pp. 31-37

11) Hay edición española Los principios de la filosofía, Madrid, Reus, 1925. La cuarta parte dedicada a la Tierra ocupa las páginas 251-284. Otra edición reciente en Alianza Editorial.

11 Ibidem, pp. 281-284: Descartes expuso que la Tierra había sido un astro como el sol, compuesto de la misma materia aunque de menor tamaño. Más tarde, había abandonado su primitivo emplazamiento y se había precipitado hacia el sol, sufriendo un proceso de enfriamiento que no había afectado a su parte central, donde había subsistido una materia comparable a la solar. En su opinión, se podían diferenciar tres regiones en la Tierra. La primera era el núcleo central $\mathbf{I}$, compuesto por una materia de igual naturaleza que la del sol. Alrededor de este núcleo I se disponía una segunda región $\mathbf{M}$, ocupada por un cuerpo muy opaco y denso. En estas dos regiones internas de la Tierra $\mathbf{I}$ y $\mathbf{M}$ el hombre no había entrado nunca. Por último, se encontraba la tercera región $\mathbf{A}$, en donde se originaban todos los cuerpos naturales que se podían encontrar en el exterior del planeta.

Al precipitarse el astro que constituía la Tierra hacia el sol, las dos regiones internas $\mathbf{I}$ y $\mathbf{M}$ no habían sufrido cambios, pero sí lo había experimentado su región más externa $\mathbf{A}$. En esta última se empezaron a formar distintos cuerpos, debido a la actividad de fenómenos físicos como la gravedad, la luz, el calor y el movimiento, lo que había dado origen a que el cuerpo $\mathbf{A}$ se dividiera en otros dos $\mathbf{B}$ y $\mathbf{C}$. El primero era un cuerpo raro, fluido y transparente, mientras que el segundo era denso, oscuro y opaco. Entre los dos anteriores se había formado un tercer cuerpo, $\mathbf{D}$, que al principio era tenue pero fue espesándose con el tiempo. Por encima de $\mathbf{D}$ se formó otro cuerpo, $\mathbf{E}$, de constitución dura y, por tanto, distinta a las de $\mathbf{B}$ y $\mathbf{D}$, que eran cuerpos fluidos. El amplio espacio entre $\mathbf{D}$ y $\mathbf{E}$ se llenó con partículas del cuerpo $\mathbf{B}$, que atravesando los poros de $\mathbf{E}$, formaron el cuerpo $\mathbf{F}$. El cuerpo $\mathbf{E}$, más pesado y denso que $\mathbf{F}$, se suspendió entre este último y el $\mathbf{D}$, constituyendo una especie de bóveda, en la que se fueron formando grietas cada vez más grandes, hasta que la bóveda ya no pudo sostenerse y cayó en pedazos por la fuerza de la gravedad sobre el cuerpo C. Algunos de los fragmentos cayeron de lado y quedaron apoyados unos contra otros. El cuerpo D, fluido y ligero, pasó a ocupar las cavidades inferiores dejadas por los fragmentos de $\mathbf{E}$, las grietas y poros, elevándose por encima de los fragmentos más bajos.

Descartes explicaba a continuación cómo se habían formado en la superficie terrestre los accidentes geográficos, como montes, llanuras y mares. Los cuerpos $\mathbf{B}$ y $\mathbf{F}$, decía, estaban formados por aire; $\mathbf{C}$, la corteza terrestre interna, era un cuerpo muy grueso; $\mathbf{D}$ estaba constituido por agua y, por último, $\mathbf{E}$, cra la superficie de la Tierra, compuesta de piedras, arcilla, arena y limo. Los mares se habían formado por el agua que había ascendido desde el cuerpo $\mathbf{D}$ entre los espacios de $\mathbf{E}$. Los fragmentos que se encontraban suavemente inclinados y que no se hallaban cubiertos por las aguas, habían constituido las llanuras, 
tratadas por el filósofo francés en su interpretación mecanicista del origen de la Tierra influyeron posteriormente en teorías de la Tierra, cuyos autores introdujeron la idea de un hundimiento de la corteza terrestre, idea cuyas raíces se encontraban en la hipótesis cartesiana.

\section{El diluvio, fenómeno de alteración y cambio geológicos.}

Las raíces del diluvismo geológico del XVII se encuentran en una disertación ${ }^{12}$ escrita a mediados de siglo por el español José Antonio González de Salas (1588-1651). Filólogo próximo al neoestoicismo ${ }^{13}$, González de Salas fue el autor que atribuyó por primera vez al diluvio una acción decisiva en el modelado del relieve terrestre. En la traducción realizada por González de Salas del Compendio Geographico i Historico de el Orbe Antiguo... (1644) de Pomponio Mela, criticaba la tesis de Esdras de que en el tercer día de la Creación las aguas, al retirarse, habían pasado a ocupar sólo la séptima parte del globo terrestre, dejando como tierra firme las otras seis partes del mundo. Esto se oponía, según Salas, al parecer de Aristóteles, quien, al desconocer la existencia del continente americano, mantenía que las aguas ocupaban diez veces más espacio que las tierras. Salas consideraba ridículas las opiniones de ciertos "filósofos de Asiria" sobre la primitiva forma redonda y perfecta de la Tierra, que carecía de las desigualdades debidas a montes y valles, o la del obispo de Siria Moses Bar-Kepha (siglo X) ${ }^{14}$ en su obra De Paradiso, para quien el paraíso y la tierra habitada por el hombre se encontraban a un lado del océano, mientras que en el otro había extensos espacios de tierra deshabitada. Durante la catástrofe diluvial, el arca de Noé había surcado el océano hasta los montes de Armenia, situados en las tierras que se hallaban fuera de los límites del paraíso.

González de Salas sostenía en su trabajo que, tras el diluvio, la Tierra emergida al retirarse las aguas era diferente de la que apareció en el tercer día de la Creación:

"Que la Tierra toda, que, después de haberse retirado la inundación de el mar en el Diluvio, apareció descubierta, para que habitación hubiese de ser de el segundo Padre, i de su larga sucesión, que en ella aún permanece, no es la misma, sino otra diferente de aquella plaga de Tierra, que recogiéndose el mar, como se ha dicho,

\footnotetext{
mientras que los más elevados originaron las montañas. Los trozos de fragmentos desprendidos al chocar éstos entre sí habían constituido las rocas costeras y las eminencias rocosas de las montañas.

12 Gonzalez de Salas, J.A. (1650), De duplici viventium terra dissertatio paradoxica, Lugduni Batavorum.

13 Sobre J. A. González de Salas puede verse Lopez RuEDA, J. (1975), "Joseph Antonio González de Salas, un filólogo clásico amigo de Quevedo" en Tres grandes humanistas españoles, Madrid, pp. 3762 y CAPEL, H. (1985), La física sagrada, Barcelona, Serbal, pp. 85-94.

14 CAPEL (1985), p. 88, nota 16.
} 
quedó aparecida, i manifiesta en el día tercero de la Creación de el Universo, i que habitación fue de los hombres antes, hasta su ruina universal; porque también hubo de ser universal para la misma Tierra, quedando desde entonces cubierta de las aguas de el mar para siempre"15.

Concebía González de Salas una estructura geodinámica de la superficie terrestre en la que la alternancia entre tierra y agua había sido una constante a lo largo de la historia de la Tierra. Estos cambios geológicos eran mencionados por autores clásicos, como Estrabón, Plinio, Eratóstenes, Hiparco y Posidonio ${ }^{16}$, citados por él. En este sentido, González de Salas pensaba que la emersión de América había tenido lugar debido a que la inundación y consiguiente desaparición de la Atlántida, isla mencionada en el Timeo de Platón, se había visto compensada por la aparición del continente americano ${ }^{17}$.

Las opiniones de González de Salas fueron rebatidas por el polígrafo José Pellicer de Ossau y Tovar $^{18}$, quien pensaba que tras el diluvio universal no se había dado una mutación general ni cambios entre continentes y océanos. En su Epistola cuyo argumento es la defensa de la Tierra única, carta que escribió a González de Salas en 1646, Pellicer mantenía que la única diferencia que había existido en la Tierra tras el diluvio era la manifestada hacia el año 350 por Ephrem, diácono de Edesa ${ }^{19}$. Del mismo parecer, decía Pellicer, habían sido Moses Bar-Kepha y en general los Padres de las Iglesias griega y latina, "que no hablaron de mutación de tierra en agua ni de agua en tierra"20. El testimonio de Ephrem, seguía Pellicer, había sido utilizado también por George Sincello, cronógrafo griego del siglo VIII y coadjutor del patriarca de Constantinopla, para acusar de erróneas las opiniones de Beroso el caldeo y Manethon el egipcio. Pellicer, casi un milenio después, utilizaba los mismos argumentos que Ephrem y Sincello, el cambio de región, para oponerse a la tesis del cambio en la distribución de tierras y aguas, propuesta por González de Salas, quien insistía en que la primera Tierra, habitada por el hombre desde Adán hasta Noé, se

15 GonZalez de Salas, J.A. (1644), "Dissertación de la Tierra descubierta i cubierta de las aguas" en Pomponio Mela Compendio Geographico i Historico de el Orbe antiguo..., Madrid (2a edición de 1780), pp. 14-61; p. 16

16 GONZALEZ De SAlas (1644) "Dissertacion de la Tierra...", pp. 55-56.

17 Ibidem, p. 58: "Inundose pues esta Isla [la Atlántida], i lo que parece mas conforme al curso de la Naturaleza; que llevamos ia aqui apprehendido, en vez de Ella se restituió despues otra tanta Tierra habitable, que (segun mi opinion) fue la America misma..."

18 Pellicer De OsSau y TOvar, J., "Epistola cuyo Argumento es la Defensa de la Tierra unica", escrita en 1646 y publicada en la Bibliotheca formada de los libros, i obras publicadas de Don Joseph Pellicer de Ossau y Tovar, Valencia, 1671, pp. 31-34.

19 Pellicer (1671), Bibliotheca..., p. 31: "Que los mortales después del Diluvio no mudaron de Tierra, porque fue la misma, sino de Territorio. Porque fue diferente la región en que habitó Noé, con sus descendientes, que la que vivió Adán con los suyos hasta Noé"

20) Ibidem, p. 31 
había convertido en mar debido a la catástrofe diluvial, mientras que lo que antes del diluvio había sido mar era en la actualidad la tierra habitada por el género humano desde que Noé desembarcara del arca ${ }^{21}$.

La versión de la disertación de González de Salas traducida al latín, De Duplici viventium terra dissertatio paradoxica (Leiden, 1650), tuvo gran repercusión en el extranjero, siendo refutada por autores que, al comentar la historia del Antiguo Testamento, sostuvieron que la disertación de Salas había inspirado la Theoria Sacra Telluris (1681) de Thomas Burnet, obra considerada la primera teoría diluvista de la Tierra 22 .

La importancia dada por González de Salas al diluvio universal, fenómeno al que atribuyó el nuevo modelado de la superficie terrestre, cambiando el primitivo relieve surgido tras la Creación, abriría una vía, principalmente en la Europa protestante, que conduciría a la emisión de las diversas teorías diluvistas de la Tierra.

\section{Las teorías cosmogónicas diluvistas en Gran Bretaña.}

La casi totalidad de los autores del siglo XVII que se interesaron por la geogenia, buscaron una explicación al estado de la corteza terrestre en la Biblia, centrándose en los efectos geomorfológicos que habían podido ocasionar el diluvio universal, e incluso, como ocurrrió en el caso de los naturalistas británicos, postulando una teoría diluvista de la Tierra.

La inundación universal bíblica cobró en Gran Bretaña desde finales del siglo XVII una importancia decisiva a la hora de explicar el relieve terrestre, dando lugar a la lectura de varias memorias sobre el tema en las Philosophical Transactions de la Royal Society de Londres ${ }^{23}$. De todas formas, fueron fundamentalmente las obras de Thomas Burnet (circa 1635-1715), de John Woodward (1665-1722) y de William

21 Ibidem, p. 34.

22 Chistiano Schottano refutó a González de Salas en una carta de 1654 publicada en su Bibliotheca historiae veteris testamenti, 1662. LOPEZ RUEDA (1975), p. 60 comenta que el teólogo luterano Johann Franz Buddeo (1667-1729), profesor en Halle y Jena, en su Historia Eclesiastica veteris Testamenti (1709) rebatió la obra de Thomas Burnet Telluris Theoria Sacra (1681), que consideraba basada en las ideas de González de Salas. David Clément, en su Bibliothèque curieuse, historique et critique (1760), t. IX, pp. 238-240, reseñó la obra de González de Salas y comentó que el filólogo W.E. Tentzel (16591707), en sus Exercitationes selectae (1692), t. I, p. 291, la mencionó como una obra original que Burnet copió en su Theoria Sacra Telluris. Tentzel comentaba que Buddeo deseaba refutar a Burnet y probar que éste era un plagiario que no había inventado la ficción de una Tierra diferente a la que existía antes del diluvio, ya que consideraba que la idea de Burnet se basaba en gran parte en la obra de González de Salas.

23 Véase STOKES, E. (1969), "The Six Days and the Deluge: some Ideas on Earth History in the Royal Society of London 1660-1775", Earth Science Journal, 3, pp. 13-39. 
Whiston (1667-1752), las que originaron una gran controversia ${ }^{24}$. Estos autores anglosajones de finales del siglo XVII, desde diferentes puntos de vista, coincidieron en interpretar que la forma actual de la corteza de la Tierra era resultado de la acción del diluvio.

La primera obra diluvista fue la de Thomas Burnet ${ }^{25}$, pastor de la iglesia anglicana. Su idea básica era que la historia pasada y futura de la Tierra se podía describir como un ciclo compuesto por las diferentes fases por las que había pasado y debería pasar el globo terrestre: el caos, el paraíso, el diluvio, el estado actual, la conflagración por el fuego y el milenio. En una de tales fases, la Tierra se había visto inundada por el diluvio universal, cuyas aguas posteriormente se habían retirado, apareciendo la superficie terrestre tal como se encontraba en el presente.

Burnet partía de que en la primera fase o estado, la Tierra había sido una esfera compuesta de una mezcla líquida de todos los elementos, los cuales se habían ido separando según su peso específico. Los elementos líquidos habían formado dos capas, una inferior de agua y otra superior de líquidos oleaginosos. Las partículas de polvo de la atmósfera se habían depositado en la capa superior aceitosa, constituyendo una corteza superior, esférica y regular, por encima del agua. Tal había sido el estado de la Tierra antes del diluvio. El calor solar y los vapores del agua subterránea agrietaron la corteza superior que se hundió en la capa de agua. El diluvio tuvo lugar debido a este hundimento, ya que al agrietarse y resquebrajarse la corteza terrestre, surgió violentamente del interior de la Tierra el agua del océano subterráneo que inundó toda la superficie. Posteriormente, el agua había vuelto a su sitio, mientras que los restos de la corteza superior, amontonados de forma irregular, formaron los actuales accidentes geográficos ${ }^{26}$.

El modelo de globo terráqueo descrito por Woodward establecía que la corteza terrestre se componía de capas horizontales, que accidentalmente se encontraban inclinadas en agunos puntos. La Tierra primitiva había sido enteramente disuelta por las aguas del diluvio, fenómeno que describía como un hundimiento de la corteza terrestre, y la materia que la componía se había precipitado en el inmenso reservorio interior de las aguas, al que Woodward denominaba gran abismo. Este abismo se comunicaba con los océanos a través de conductos interiores. El motor del sistema era el calor interno del globo.

\footnotetext{
24 BURNET, T. (1681), Telluris Teoria Sacra, orbis nostri originum et mutationes generales, London; Woodward, J., (1695) An Essay toward a Natural History of the Earth and terrestrial Bodies, London y WHISTON, W. (1696), Theory of the Earth, London.

25 Acerca de las ideas de Burnet puede verse Gould, S.J. (1987), Time's Arrow Time's Cycle. Myth and Metaphor in the Discovery of Geological Time, Harvard University Press (Edición española La flecha del tiempo, Madrid, Alianza, 1992. Sobre Burnet, especialmente las páginas 39-68).

26 Cf. ROGER (1973), pp. 39-40.
} 
Whiston, por su parte, fue partidario de que el diluvio había sido provocado por un cometa. Whiston fue sucesor de Newton en la cátedra de Cambridge, pero su maestro se distanció de él cuando adoptó posturas radicales en cuestiones religio$\operatorname{sas}^{27}$.

La tesis que adoptó Whiston fue que en tiempos del diluvio había pasado cerca de la Tierra un cometa con una larga cola formada por vapores rarificados, los cuales se habían condesado, cayendo en forma de lluvias abundantes durante cuarenta días. $\mathrm{Al}$ mismo tiempo, el impacto oblicuo o la atracción del cometa había desplazado el eje de los polos, transformando la primitiva órbita circular en otra elíptica y provocando un aumento en la fuerza de las mareas, en particular las de las aguas subterráneas del océano interior, que surgieron e inundaron la superficie terrestre. El peso de las aguas originó el hundimiento de la corteza en distintos puntos, originándose así la inundación universal.

Entre los miembros de la Royal Society que discutieron sobre las causas del diluvio se encontró el astrónomo Edmond Halley (1656?-1743) ${ }^{28}$. Halley sugirió que el choque accidental de un cometa o de algún otro cuerpo celeste que pasó cerca de la Tierra, había dado lugar a un cambio repentino en los polos y el eje de la Tierra, de manera que el mar se retiró de aquellos lugares hacia los cuales los polos se aproximaron y cubrió las regiones de donde los polos se apartaron. El choque había obligado a las aguas a desplazarse repentinamente hacia el lugar del globo terrestre que recibió el impacto del cometa. La fuerza de este impacto había sido lo suficientemente grande como para hacer salir al océano de su lecho y desplazarlo sobre los continentes ${ }^{29}$.

Por último, la respuesta al problema de la importancia del diluvio en el modelado del relieve terrestre que emitió Robert Hooke (1635-1703), conservador y posteriormente secretario de la Royal Society de Londres, se conoce a través de Halley ${ }^{30}$. Hooke imaginaba la Tierra como una especie de cáscara oval que había sido comprimida hasta formar un esferoide alargado, lo cual provocó que salieran las aguas del abismo interior. A Halley le parecía que esta hipótesis no se basaba en las leyes de la naturaleza, por lo que pensaba que sólo podía sostenerse recurriendo al "dedo sobrenatural de Dios", quien había comprimido el globo terrestre y posteriormente lo había restituido a su antigua forma.

27 Acerca de William Whiston puede verse CASINI, P. (1971), El Universo máquina. Orígenes de la filosofía newtoniana, Barcelona, Martínez Roca, pp. 91-116.

28 Halley leyó sendas memorias sobre el diluvio el 12 y el 19 de diciembre de 1694: "Of the cause of the universal deluge. Written by Dr. E. Halley", Philosophical Transactions, 33, $\mathrm{n}^{\circ}$ 383, May 1724, pp. 118-124.

29 Bibliotheque angloise, t. XII, p. 344.

30) Ibidem, pp. 342-343. 


\section{La Protogaea (1693) de G. W. Leibniz.}

Una interpretación del origen y formación de la corteza terrestre, que admitía la acción geológica del diluvio, fue propuesta a finales del siglo XVII por G. W. Leibniz. Las ideas geológicas expuestas por Gottfried Wilhelm Leibniz (1646-1716) han sido objeto de un análisis bastante detallado ${ }^{31}$. En su interés por el estudio de la historia de la Tierra influyeron varios factores: la lectura de la obra del geólogo danés Niels Stensen o Nicolaus Steno (1638-1686), el encargo del duque Ernst-August en 1680 de que escribiera la historia de la casa de Hannover y del ducado de Brunswick y sus visitas a las minas de las montañas del Harz, entre 1680 y 1686 . La convergencia de tantas circunstancias facilitó el que Leibniz pudiera exponer su hipótesis sobre el origen de la Tierra, en un resumen publicado en las Acta eruditorum de Leipzig en 1693 con el título de Protogaea Auctore G.G.L. ${ }^{32}$.

31 Sobre las ideas geológicas de Leibniz puede consultarse PeCAUT, C. (1951), "L'oeuvre géologique de Leibniz", Revue Générale des Sciences, t. LVIII, pp. 282-296; STICKER, B. (1967), "Leibniz' Beitrag zur Theorie der Erde", Sudhoffs Archiv fur Geschichte der Medizin und der Naturwissenschaften, Band 51, Heft 3, pp. 244-259 y RoGeR, J. (1968), "Leibniz et la théorie de la Terre", Journées Leibniz organisées au Centre International de Synthèse. 1966, pp. 137-144.

32 "PROTOGAEA AUTORE G.G.L." Acta eruditorum anno MDCXCIII publicata, Leipzig, 1693, pp. 40-42. Una traducción inglesa fue publicada por OLDROYD, D.R. and HOWES, J.B. (1978), "The first published version of Leibniz's Protogaea", J. Soc. Biblphy Nat. Hist., 9 (1), pp. 56-60:

"Estas ideas fueron inspiradas por una investigación sobre la naturaleza de las regiones situadas entre las montañas Hercinianas y el Océano. El autor [Leibniz se refiere a sí mismo en tercera persona] cree que el globo terrestre ha sufrido cambios mucho mayores de lo que se podría suponer. Primero, propone que la mayor parte de su materia ha sido producida por el fuego, particularmente en la separación general de la luz de las tinieblas descrita por Moisés. Y de ello debemos inferir - como algunos creen - que los cuerpos oscuros o planetas (entre los cuales consideran que debería incluirse la Tierra) se formaron a partir de [cuerpos] fijos o brillantes, es decir, estaban cubiertos de "puntos", como por escoria producto de una conflagración. El autor cree que la corteza era un tipo de vitrificación: y así la base de la Tierra es vidrio, cuyo fragmentos son las arenas; y más tarde se formaron los diferentes tipos de tierra por la mezcla de sales y la circulación de aguas y vapores. Más aún, el autor cree que el humor expulsado al aire por acción del fuego se enfrió posteriormente y se condensó formando una corteza, más o menos de la misma forma que los objetos calcinados atraen la humedad y así se formó la categoría de lixivia [lejía, una solución de carbonato potásico] o (empleando la expresión química) la categoría de oleum per deliquium [potasa licuada]. Y esto dió origen al mar, que está cargado de sal fijada por el lavado del empyreuma de la superficie terrestre; del mismo modo que el santo Moisés postulaba la separación de la luz y la oscuridad, es decir, la separación del principio activo del fuego de los demás elementos más pasivos. Luego, por la separación de los cuerpos pasivos, que variaría en función de su grado de resistencia, es decir, de los fluidos y de los sólidos, dividió las aguas de la tierra. Más aún, el autor piensa que el mar cubría en su momento una gran parte de la Tierra que ahora aparece al descubierto y que en un momento alcanzó las cumbres de las montañas; hasta que la corteza de la Tierra, hueca toda ella, se partió por su propio peso y por el de las aguas y posiblemente también por terremotos. Por esto se encuentran estratos inclinados rotos en las regiones montañosas, que suelen estar llenos de conchas, glossopetras y otros 
En su Essai de Theodicée (1710), Leibniz comentó el origen y la historia de la Tierra. Al referirse a la existencia de monstruos en la naturaleza y anomalías en el universo, exponía que lo único que se conocía del globo terrestre era su superficie, ya que apenas se había penetrado en su interior. En la corteza terrestre se podían apreciar las huellas de las grandes revoluciones que la habían afectado en el pasado. Insistía en su tesis de que la Tierra estuvo en algún momento del pasado en ignición y que las rocas que constituían la base de la corteza terrestre eran las escorias resultantes de una gran fusión. En su interior se encontraban metales y minerales parecidon a los que se podían extraer de los hornos de fundición. El mar, por su parte, era en su totalidad una especie de oleum per deliquium. Tras el enfriamiento posterior al incendio, la humedad que el fuego había elevado al aire volvió a caer sobre la superficie terrestre, lavándola al tiempo que disolvía y arrastraba la sal que había quedado entre las cenizas, hasta llenar las cuencas de los océanos con agua salada. Tras el fuego, habían operado la tierra y al agua, que también habían contribuido a modelar la superficie terretre. Posteriormente, la corteza, formada por el enfriamiento y con grandes cavidades bajo ella, se había hundido, de manera que según algunos autores, como T. Burnet, vivíamos sobre las ruinas de un mundo anterior. Muchos diluvios e inundaciones habían dejado sus sedimentos y sus huellas indicaban que el mar había ocupado en el pasado lugares que hoy eran tierra firme. Tras cesar estos cataclismos, el globo terrestre había tomado la forma que tenía en el presente. Según Leibniz, el Génesis recogía estos grandes cambios geológicos, y así la separación de la luz y de las tinieblas indicaba la fusión causada por el fuego, mientras que la separación de lo húmedo y de lo seco señalaba los efectos de las inundaciones ${ }^{33}$.

\begin{abstract}
restos dejados por el mar tras su retirada, atrapados en el fango, que enseguida se endureció. Y piensa que el mar que había cubierto previamente las montañas penetró luego por las grietas abiertas en el interior de los abismos y así una gran parte de la Tierra quedó al descubierto. Cree que otros fenómenos pueden atribuirse no sólo a un diluvio universal sino también a diversas y enormes inundaciones locales. Además, entre otros indicios del fuego no sólo ve la sal del mar, sino también las numerosas obras subterráneas de la naturaleza, que son análogas a los resultados de los experimentos llevados a cabo en los laboratorios químicos y que se han de atribuir a la fusión volcánica, sublimación, solución y precipitación. El autor considera que la sedimentación acuosa queda probada por los estratos que componen la Tierra y por las cosas contenidas en ella, las cuales fueron arrastradas por el mar o por la arena, así como por las formas de los cuerpos que han quedado congelados por algún tipo de cristalización. Dice que hace falta cautela para distinguir los efectos del fuego y del agua, porque en la naturaleza se producen casi los mismos efectos tanto por un proceso húmedo como por otro seco, y las cosas que se han producido por un enfriamiento tras la fusión o sublimación, han tomado sus formas del mismo modo que lo han hecho áquellas que han sido coaguladas tras una solución y precipitación."

3. LEIBNiz, G.W. (¿1928?), La Teodicea o Tratado sobre la libertad del hombre y el origen del mal, Madrid, M. Aguilar, pp. 293-294.
\end{abstract}




\section{LOS SISTEMAS GEOLÓGICOS DILUVISTAS EN EL SIGLO XVIII.}

El diluvio universal fue durante el siglo XVIII el punto de referencia geológico necesario para todos aquellos naturalistas interesados en explicar el pasado de la Tierra y de la vida sobre ella ${ }^{34}$. Con este fenómeno podían explicarse conjuntamente el modelado histórico del relieve terrestre y el registro de invertebrados marinos fósiles, pero también servía para resolver el problema del enterramiento de grandes mamíferos exóticos, en regiones climáticas muy diferentes de su hábitat natural.

Tal y como aparecía en el Génesis, el relato del diluvio universal exponía en síntesis que la Tierra había sido inundada por las aguas que procedían de las cataratas del cielo y de las fuentes del abismo. Al ser una exposición tan ambigua no es de extrañar que en torno al diluvio surgieran discrepancias en cuestiones relativas a su universalidad, salinidad etc., que dieron lugar a algunas polémicas ${ }^{35}$, principalmente, aunque no sólo, entre teólogos estudiosos de la exégesis bíblica. En primer lugar se discutía si todos los peces, tanto de agua dulce como los marinos, habían sobrevivido. Esto era una consecuencia del debate sobre la salinidad de las aguas del diluvio, o dicho de otra manera, sobre la procedencia de las mismas. Si venían de las "fuentes del abismo" —el relato bíblico no aclaraba nada sobre donde se encontraban estas "fuentes" —o de lluvias intensas, el agua tenía que ser dulce, pero al caer en mares y océanos éstos se habrían desbordado y cubierto las tierras. Según esto, al mezclarse los dos tipos de agua, saladas y dulces, las aguas de la inundación tenían que haber sido saladas, aunque con menor proporción de sal que las marinas.

Durante las primeras décadas del siglo XVIII, en Suiza y en algunos estados alemanes, la hipótesis diluvista de los fósiles fue la explicación más socorrida ${ }^{36}$. El relevo de las teorías diluvistas de la Tierra, propuestas a finales del XVII por Burnet, Whiston y Woodward fue recogido en Suiza por los hermanos Scheuchzer y por L. Bourguet.

\footnotetext{
34 Un panorama histórico de la geología en el siglo XVIII puede encontrarse, además de las obras ya citadas, en: OLDROYD, D.R. (1979), "Historicism and the Rise of Historical Geology", History of Science, XVII, pp. 191-213 y 227-257 y ELLENBERGER, F. (1994), Histoire de la Géologie. La grande éclosion et ses prémices 1660-1810, Paris, Technique et Documentation.

35 Cf. BlignY, M. (1973), "Il mito del diluvio universale nella coscienza europea del seicento", Rivista Storica Italiana, LXXXV, (1), pp. 47-63; Para el debate sobre el diluvio en Europa durante el siglo XVIII puede verse ELLENBERGER, F. (1978), "Le dilemme des montagnes au XVIII ${ }^{\text {imk }}$ siècle: vers une réhabilitation des diluvianistes?", Revue d'Histoire des Sciences, XXXI, pp. 43-52 y RAPPAPORT, R. (1982), "Geology and Ortodoxy: the case of Noah's fload in Eighteenth-Century thought", The British Journal for the History of Science, XI, pp. 1-18.

36 En relación a la literatura diluvista en Suiza pueden consultarse las obras de CAPEL (1985), pp. 112-120 y La teoría física de la Tierra. Una tesis en la Ginebra del siglo XVIII, Barcelona, Geo-crítica, 1982.
} 
Johannes Scheuchzer (1684-1738), en una memoria leída en la Academia de Ciencias de París ${ }^{37}$, expuso que Dios, tras el diluvio universal, queriendo que las aguas retornaran a los reservorios subterráneos, había quebrado y dislocado con su todopoderosa mano un gran número de lechos terrestres, dispuestos anteriormente de forma horizontal, y los había levantado sobre la superficie terrestre, formándose las montañas. Como éstas tenían un núcleo muy duro, Scheuchzer señalaba que Dios sólo había actuado en los lugares donde existían muchos sedimentos de piedra. De aquí que los países que como Suiza tenían muchas de estas capas fueran muy montañosos, en cambio otros como Flandes, Alemania, Hungría y Polonia, cuyo suelo sólo se componía de arenas y arcillas incluso a una gran profundidad, carecieran prácticamente de montañas.

En una reunión posterior de la Academia, en 1710, Scheuchzer explicó el diluvio suponiendo que si Dios, en un momento dado, hubiera parado el movimiento giratorio de la Tierra sobre su eje, las aguas del mar hubieran inundado violentamente todos los continentes ${ }^{38}$.

Una idea de la importancia geológica del diluvio la puede dar el hecho de que el hallazgo de unos huesos supuestamente humanos en Oeningen llevó a considerar a Johann Jacob Scheuchzer (1672-1733) ${ }^{39}$, hermano del anterior, que eran los restos de una persona ahogada durante la inundación universal. Así se lo hizo saber al Secretario y posterior Presidente de la Royal Society de Londres, Hans Sloane, en una carta de finales de 1725, el hallazgo del esqueleto de un Homo diluvii testis, u hombre testigo del diluvio, que corroboraba la catástrofe diluvial ${ }^{40}$.

37 Histoire et Mémoires de l'Académie des Sciences..., 1708, pp. 30-33.

38 Ibidem, 1710, pp. 19-21.

39) Johann Jacob Scheuchzer, medico de Zurich, publicó la traducción latina de la obra de Woodward Specimen Geographica Physicae (1704) y posteriormente obras de carácter diluvista Piscium querelae ef vindiciae (1708), Herbarium Diluvianum (1709), Musaeum diluvianum (1716) у Physica $\operatorname{sacra}(1731)$.

40 La carta de Scheuchzer a Sloane se publicó en las Philosophical Transactions, t. XXXIV, En.Feb. 1726, $\mathrm{n}^{\circ}$ 392, pp. 38-39. Scheuchzer difundió por Europa la información sobre los supuestos huesos humanos diluviales, comunicando también el hallazgo al presidente de la Académie des Sciences de París, al abad Jean Paul Bignon (1662-1743): "Extrait d'une lettre ecrite de Zurich à M. l'Abbé Bignon, par M. Scheuchzer, Docteur en Medecine etc., au sujet des restes d'un homme noyé dans le Deluge Universel", carta publicada en el Journal des Sçavants, juin 1726, pp. 378-379, y envió la misma información a la revista Sammlung von Natur-und Medicin-Geschichten, en donde se publicó la nota con la descripción de los huesos, en abril de 1726 


\section{El primer intento de clasificación de teorías de la Tierra.}

Contemporáneo de Scheuchzer fue Louis Bourguet (1678-1742) ${ }^{41}$, autor de unas Lettres philosophiques (1729), en donde publicó su "Mémoire sur la theorie de la Terre"42. En esta memoria exponía que la elaboración de una teoría de la Tierra podía considerarse como una ciencia nueva, que deducía los fenómenos naturales y la formación del globo terrestre, a partir de los cambios ocurridos en el pasado y los que aún quedaban por llegar.

Según Bourguet, todas las opiniones que se habían emitido sobre la teoría de la Tierra podían enmarcarse en tres hipótesis. La primera hacía referencia al hundimiento o caída -entendida ésta tanto en el sentido moral como en el físico- del antiguo mundo, es decir, la disolución de la primitiva tierra surgida en la creación. Había sido propuesta por Thomas Burnet (1681), aunque anteriormente ya había sido sugerida por Platón, de quien la había tomado Francesco Patrizi (1529-1597)43. También José Antonio González de Salas había expuesto esta hipótesis, aunque, decía, con otra perspectiva.

La segunda hipótesis planteaba que el mar había ocupado en el pasado las regiones que en la actualidad formaban los continentes, de donde se había retirado lentamente. Sus raíces se encontraban en los escritos de clásicos como Aristóteles, Estrabón, Jantos, Eratóstenes y Plutarco, y había sido propuesta por Leibniz (1693) y otros naturalistas ${ }^{44}$. Una variante de esta segunda hipótesis, que tomaba algunos aspectos de la primera eran, según Bourguet, las teorías de Steno, Whiston, Halley, etc..$^{45}$.

41. Sobre L. Bourguet puede verse BORK, K. B. (1974), "The Geological Insights of Louis Bourguet (1678-1742)", Journal of the Scientific Laboratories, Denison University, vol. 55, pp. $49-77$.

42 Bourguet, L. (1729), Lettres philosophiques, Amsterdam, pp. 177-220.

4.3 Bourguet citaba en este sentido la obra de Patrizi Dialoghi della Rhetorica della Antichi, Venecia, 1562.

44 Bourguet citaba en este apartado a Alessandro de Alessandri (1461-1523), con su obra Genialum Dierum (1532); Andrea Cesalpini (1519-1603) autor de De metallicis (Roma 1596); Girolamo Fracastoro (ca. 1478-1553); Fabio Colonna (1616); Agostino Scilla (1629-1700), autor de La vana speculazione disingannata dal senso (Napoles, 1670); Paulo Boccone (1633-1704) y sus Recherches \& observationes naturelles (Amsterdam, 1674) y Museo di fisica e di esperienze variato (Venecia, 1697); Antonio Vallisneri (1721) y las disertaciones de Antoine de Jussieu (1718), René-Antoine F. de Reaumur (1720) y Jean Jacques d'Ortus de Mairan (1678-1771) (1721), leídas en la Académie des Sciences de París.

45 Bourguet citaba en este apartado varias obras de Nicolaus Hartsoeker (1656-1698): Principes de physique (Paris, 1696), Conjectures physiques (Amsterdam, 1706), Suite des "Conjectures physiques" (Amsterdam, 1708) y Eclaircissements sur les "Conjectures physiques" (Amsterdam, 1710); a David Büttner y su Rudera diluvii testes (Leipzig, 1710), a Henry Gautier (1660-1737), autor de "Nouvelles Conjectures sur le globe de la terre", publicada en la Bibliothèque des Philosophes et des Sçavants (1723) y a Louis Bertrand Castel (1722). 
La tercera hipótesis, que consideraba la "disolución del primer mundo" en las aguas del diluvio universal, había sido propuesta por los diluvistas más radicales como Woodward, Scheuchzer y Joseph Monti (1682-1760), profesor en la Universidad de Bolonia.

Esta clasificación sería publicada posteriormente por un compatriota de Bourguet, Elie Bertrand (1713-1797), quien en su Mémoires sur la structure intérieure de la Terre (Zurich, 1752) añadía entre los partidarios de la segunda hipótesis a Carl von Linné, B. de Maillet y Buffon. Esta memoria de Bretrand sería traducida al castellano a finales del siglo XVIII ${ }^{46}$.

\section{La crítica a las teorías diluvistas.}

El gran número de problemas que arrastraba cualquier explicación geológica diluvista provocaron que algunos autores terminaran remitiendo al carácter milagroso de la inundación, con lo cual pretendían zanjar definitivamente la cuestión, pero lo que hacían era complicarla aún más, ya que se entraba en un nuevo debate: si la catástrofe diluvial había sido o no un fenómeno natural. Esta polémica sobre el carácter milagroso o natural del diluvio estuvo muy ligada a la influencia que la ortodoxia religiosa ejerció sobre los naturalistas que discutieron esta interpretación. En general, se puede hablar de dos situaciones distintas, según que los autores pertenecieran al ámbito protestante o católico. Mientras que los primeros, como hemos visto en Gran Bretaña, Suiza o los estados protestantes alemanes, no tuvieron ningún reparo en buscar una explicación racional del relato bíblico, los católicos, al no poder efectuar una interpretación libre de las Escrituras, fueron por lo general más prudentes y tendieron a explicar el diluvio como un milagro.

En favor del carácter natural del diluvio se aportaban los testimonios de los clásicos griegos y romanos, que citaban inundaciones locales, como los diluvios de Deucalion y Ogiges. Sin embargo, este mismo argumento se volvió en su contra, ya que algunos naturalistas describieron el diluvio como una de estas catástrofes regionales, al considerar que una inundación general de toda la Tierra era científicamente imposible.

La integración en el debate sobre el diluvio de factores científicos, ideas filosóficas y creencias religiosos, dió lugar a que los naturalistas centroeuropeos interesados por la historia geológica de la Tierra expusieran el tema de forma muy compleja.

46 Fue publicada en el Memorial Literario Instructivo y Curioso, t. VIII, junio 1795, pp. 386-414: Ibidem, t. XII, mayo 1796, pp. 184-203 y 225-253 e Ibidem, t. XIV, octubre 1796, pp. 34-78. 
Esto se puede comprobar en las obras de autores franceses, alemanes y suizos, en las que la aceptación explícita del diluvio universal no significaba que se admitiera su importancia geológica.

\section{El rechazo al diluvismo en Francia.}

A mediados del siglo XVIII hubo un rechazo, particularmente centrado en Francia, hacia las propuestas de los autores europeos partidarios de que el diluvio era el fenómeno que explicaba de forma más general, la presencia de conchas y de restos marinos en montañas y lugares alejados del mar. La crítica se centró principalmente en que los diluvistas forzaban de alguna manera el relato bíblico del diluvio universal, al utilizarlo para explicar fenómenos puramente físicos. Los autores críticos con el diluvismo sostenían que había que limitarse a una lectura textual del Génesis y no presuponer efectos, como el depósito de conchas marinas, que no se recogía en la exposición bíblica.

En este grupo de autores contrarios a forzar el relato del diluvio para explicar la presencia tierra adentro de organismos marinos petrificados, coincidieron desde católicos ortodoxos, como el jesuita Louis Bertrand Castel (1688-1757), hasta deístas como Voltaire (1694-1778), pasando por naturalistas que empleaban métodos cercanos al positivismo en el estudio de la naturaleza, como Buffon. Todos ellos aceptaban que el diluvio universal, tal como se recogía en las Escrituras, había inundado la Tierra, pero lo restringían a un suceso ocurrido por la voluntad expresa de Dios y, como tal, su explicación se encontraba fuera de los límites de la naturaleza.

Sin embargo, algunos autores fueron un poco más lejos y se cuestionaron el mismo fenómeno del diluvio universal. Este fue el caso de Benôit de Maillet (16591738), quien encontraba problemas hidrográficos que impedían suponer una inundación natural de la Tierra.

Pero la crítica al diluvismo geológico no fue exclusiva de los naturalistas y escritores franceses. Durante los años centrales del siglo XVIII filósofos alemanes, como Samuel Christian Hollmann (1696-1787) e Immanuel Kant (1724-1804), o clérigos ilustrados, como el suizo Johann Georg Sulzer (1720-1779), se interesaron por cuestiones, en principio no muy cercanas a sus áreas habituales de trabajo, como eran las del origen de los fósiles y los cambios acaecidos en la Tierra. Todos ellos coincidieron en señalar que no podía atribuirse al diluvio un papel determinante en la historia geológica terrestre. 


\section{FRANCISCO PELAYO}

\section{La "Théorie de la Terre" (1749) de Buffon.}

Georges Louis Leclerc, conde de Buffon (1708-1778) dedicó el segundo discurso de su primer tomo de la Histoire naturelle générale et particulière (Paris, 1749) ${ }^{47}$ a describir la historia de la Tierra tal como él creía que se había desarrollado.

Su modelo se basaba en que en los primeros tiempos, tras la Creación, el globo terrestre había sufrido unas revoluciones geológicas cuya intensidad no podían compararse con la de las alteraciones acaecidas en los últimos tres mil años. Pero esta invocación a lo que parecía una aceptación catastrofista del pasado geológico de la Tierra quedaba matizada cuando pasaba a explicar su tesis sobre la formación de la corteza terrestre. Decía que las materias que componían la superficie terrestre se habían solidificado por la acción de la gravedad y demás fuerzas físicas, que habían unido las partículas de tales materias. Esto le hacía pensar que la superficie terrestre debía de haber sido en un principio menos sólida de lo que era en el presente. Por tanto, las mismas causas que en la actualidad sólo producían alteraciones débiles o casi imperceptibles operando durante muchos siglos, en los primeros tiempos habrían ocasionado enormes revoluciones en muy pocos años.

Por otra parte, decía Buffon, en tiempos del diluvio, la gravedad y demás fuerzas físicas ya habían actuado durante más de dieciséis siglos sobre la superficie terrestre, por lo que ésta habría alcanzado un grado de solidificación tal que no parecía posible que las aguas del cataclismo, en el poco tiempo que había durado la inundación universal, hubieran podido alterar profundamente el relieve de la Tierra ${ }^{48}$.

Buffon sostenía que en el pasado las aguas habían cubierto la superficie terrestre, por lo que ésta había tenido que ser por algún tiempo fondo del mar. El hecho de que el mar no hubiera permanecido sobre las tierras se debía, según Buffon, por un lado, a un fenómeno de tipo actualista, como era su movimiento diario y continuo con el que ganaba terreno en unas costas y lo perdía en otras, y por otro, a catástrofes, como las grandes inundaciones periódicas, diluvios, hundimientos de vastas cavernas subterráneas y las grandes revoluciones generales padecidas por el relieve terrestre, que habían dado lugar a la retirada del mar de las tierras que anteriormente ocupaba.

$\mathrm{Al}$ abordar las pruebas de su "teoría de la Tierra"49, Buffon comenzaba sugiriendo que nuestro planeta se habría formado a partir de un trozo desprendido del sol, al chocar con éste algún cometa. Para él esto era tan probable como la hipótesis de

47 En la edición original francesa citada en el texto el "Second Discours. Histoire et Théorie de la Terre" ocupa las páginas 65-112 del primer tomo. Para la redacción de esta páginas hemos utilizado la primera traducción española de José Clavijo y Fajardo Historia Natural, general y particular (Madrid, 1785), en la que la "Historia Teórica de la Tierra" ocupa las páginas 63-124.

48 Ibidem, t. I, pp. 76-77.

49 Ibidem, t. I, pp. 127-171. 
Leibniz de que todos los planetas eran antiguos soles. Coincidía con el filósofo alemán en su suposición de que la Tierra en algún momento estuvo en estado fluido, pero encontraba errónea la propuesta de Leibniz porque no explicaba el estado presente de la Tierra y sólo se refería a su pasado. Éste, decía, era tan remoto y había tan pocas señales del mismo que podía decirse de él cuanto se quisiese. Posteriormente, dedicaba más de treinta páginas, en la primera edición española de su obra, a discutir los sistemas geológicos de Whiston, Burnet, Woodard, Bourguet, Leibniz, Scheuchzer, Steno... De todos ellos Buffon aceptaba los fenómenos catastróficos, como inundaciones, terremotos, hundimientos..., apelados por algunos autores para explicar las desigualdades de la superficie terrestre, pero sólo como causas secundarias que habían provocado ligeras alteraciones geológicas. Él sostenía que tales causas secundarias estaban subordinadas a la causa primera, que era el movimiento de flujo y reflujo y el movimiento del mar de oriente a occidente ${ }^{50}$.

Buffon aprovechaba para dejar clara su posición contraria al supuesto del diluvio como factor de alteración geológica mundial y responsable del depósito de conchas en los montes: "...debemos considerar el Diluvio Universal un medio sobrenatural de que se valió la Omnipotencia divina para castigar a los hombres, y no como efecto natural en que todo hubiese sucedido conforme a las leyes de la Física. El Diluvio Universal es, pues, un milagro en su causa y en sus efectos." ${ }^{51}$

Como el diluvio era un hecho cierto, Buffon aceptaba que pudieran discutirse las consecuencias físicas ocasionadas por dicho fenómeno, pero había que insistir en su carácter milagroso. Y decía al respecto: "...es preciso empezar confesando que el Diluvio Universal no pudo obrarse por medios naturales, reconocerle por efecto inmediato de la voluntad del Todopoderoso, ceñirnos a no indagar de él sino solamente lo que los libros Sagrados nos enseñan, confesar al mismo tiempo que no nos es permitido saber más, y sobre todo que no mezclemos una mala Física con la pureza del Sagrado libro." 52

En el Génesis, proseguía Buffon, no se decía que se formaran montes debido al diluvio, ni que las aguas estuviesen lo suficientemente agitadas para arrastrar conchas del fondo del mar y depositarlas y esparcirlas por la tierra, ni que el globo terrestre hubiera sufrido una disolución total.

Buffon terminaba con la cuestión del diluvio diciendo que el Génesis se había limitado a exponer que este cataclismo había servido para destruir a los seres vivos, no hablando de ninguna alteración o cambio geológico de la Tierra.

\footnotetext{
50) Ibidem, p. I, p. 203.

51 Ibidem, t. I, pp. 206-207.

52 Ibidem, t. I, pp. 208-209.
} 


\section{FRANCISCO PELAYO}

\section{El artículo "Deluge" (Diluvio) de L'Encyclopédie.}

Nicolas Boulanger (1722-1759) ${ }^{53}$ y Denis Diderot (1713-1784) fueron los autores de la voz "Déluge" publicada en la Encyclopédie ${ }^{54}$. Esta entrada recogía que tres eran los puntos problemáticos en la cuestión del diluvio: su extensión — si había sido o no universal-, su causa y sus efectos. El discurso sobre el diluvio fue el siguiente:

Planteada la primera cuestión, si el diluvio había sido universal o no, Diderot comentaba que el hecho de haber sido necesaria una inmensa cantidad de agua para inundar a todo el globo terrestre había hecho suponer a varios autores que el diluvio había tenido que ser inevitablemente parcial. Sin embargo, el Génesis dejaba bien claro que el diluvio había sido universal. Además, de no haber sido universal, hubiera sido innecesario emplear cien años en construir el arca para encerrar a una pareja de animales de cada especie. Hubiera bastado con dirigirlos hacia los lugares que habían quedado sin inundar.

Una prueba que alegaban los partidarios de la universalidad del diluvio era, según Boulanger, que la tradición de un diluvio se conservaba en todos los pueblos conocidos. Pero las pruebas físicas de tales diluvios locales, que para los diluvistas se hallaban en los ángulos y declives de todos los valles de la Tierra, para la mayoría de los naturalistas sólo probaban que los mares habían cubierto los continentes, sin que esto indicara una inundación pasajera.

Para Boulanger, tampoco los organismos marinos que se encontraban en tierra adentro podían ser aportados como pruebas de los cambios geológicos ocasionados por un diluvio universal. Decía que se hallaban conchas rotas en las piedras que habían servido para construir las pirámides de Egipto y dada la inmensa antiguiedad de éstas, era díficil explicar el fenómeno sin admitir la eternidad del mundo. Explicar la presencia de conchas en las pirámides por una causa y el hallazgo de los mismos cuerpos en distintos lugares por otra, resultaba ridículo. De todas formas, decía, en las cuestiones en las que estaba la fé de por medio no había necesidad de buscar una explicación a todo. Además, estas dos pruebas, según Boulanger, eran incompatibles. Si los valles continentales eran efectos de las torrenciales aguas diluviales, los organismos fósiles no podían haber sido depositados por tales torrentes, ya que se encontraban en las zonas continentales que carecían de huellas de la acción diluvial.

$\mathrm{Al}$ abordar la segunda cuestión, la procedencia de las aguas diluviales, Diderot comentaba que autores como Steno, Burnet, Woodward y Scheuchzer habían propuestos distintos sistemas geológicos siguiendo la teoría de Descartes, quien basándose en principios mecánicos, había supuesto que en su origen el globo terrestre

53 Cf. Roger, J. (1953), "Un manuscrit inédit perdu et retrouvé: Les anecdotes de la Nature de Nicolas-Antoine Boulanger", Revue des Sciences Humaines, pp. 231-254.

${ }^{54}$ Encyclopédie, Paris, 1754, t. IV, pp. 795-803. 
había sido un cuerpo liso sin montañas ni valles. Por su parte, Boulanger criticaba a los diluvistas como su compatriota Nöel Pluche (1688-1761), y los citados Bourguet, Burnet y Whiston. De los últimos decía que se habían equivocado al recurrir para explicar el diluvio a causas físicas muy rebuscadas, cuando tenían otras más sencillas.

Boulanger discutía el problema del origen del diluvio, afirmando que la flexibilidad de las capas continuas de la Tierra era una de las principales causas del movimiento periódico que agitaba el agua de los mares en sus ensenadas. Era muy posible, decía, que la elasticidad de la bóveda terrestre, fuertemente perturbada por el diluvio, hubiera permitido que los mares se extendieran sobre los continentes, mientras que éstos eran impulsados hacia el centro de la Tierra y se sumergían bajo las aguas, en una alternancia semejante a las de las mareas, pero a una gran velocidad. De manera que el lecho de los mares tan pronto estaba en seco al inundar las aguas los continentes, como recobraba su estado natural al retirarse las aguas a su lecho original. El naturalista podía aceptar una operación de tales características y el teólogo no encontraría nada en ella contrario al texto del Génesis. Sólo habrían sido necesarias las aguas de nuestro globo terrestre sin que ningún hombre hubiera podido escaparse a tales mareas universales.

La tercera y última cuestión que se discutía en el artículo de la Encyclopédie era la que hacía referencia a los efectos del diluvio: planteaba un problema, el del cómo había tenido lugar el paso de los animales desde el continente euroasiático al americano, que en el caso de los domésticos no entrañaba dificultad, pero sí en el de los animales salvajes. A menos que se quisiera suponer una nueva creación de especies en América, algo sin fundamento, había que pensar que los dos continentes habían estado cercanos o que habían existido entre ambos pasos de tierra.

Una crítica al diluvio universal, parecida a la de la entrada Deluge de la Encyclopédie, puede encontrase en otro enciclopedista: el barón D'Holbach (1723-1789).

D'Holbach no cuestionó la realidad y universalidad del diluvio, cuyo relato, decía, se hallaba detallado en el Génesis, pero sí el error de sus partidarios, cuando atribuían sólo a esta catástrofe la formación de las capas sedimentarias y el estado actual de la Tierra. Sostenía que las Escrituras se limitaban a señalar la vía milagrosa utilizada por Dios para castigar al género humano, sin especificar nada que pudiera limitar las opiniones de los naturalistas sobre los efectos físicos que el diluvio pudiera haber ocasionado sobre el relieve terrestre.

\section{El análisis y el debate de los sistemas geológicos a fines del siglo XVIII.}

Las últimas décadas del siglo XVIII en Europa Occidental se caracterizaron desde el punto de vista de la geología por la proliferación de obras que discutían la validez 


\section{FRANCISCO PELAYO}

de anteriores propuestas sobre la historia de la Tierra y proponían nuevos sistemas geológicos.

Por un lado, no desaparecieron los autores que seguían considerando a la catástrofe diluvial como un fenómeno clave para poder explicar muchas cuestiones geológicas y paleontológicas. Estos últimos, como Johann Friedrich Esper (1732-1781) y Pyotr Simon Pallas (1741-1811), plantearon en firme la posibilidad, sugerida por algún autor como Leibniz, de la extinción de especies, recurriendo para ello al diluvio como posible explicación de lo que consideraban anomalías paleontológicas, es decir, el hallazgo de mamíferos fósiles lejos de su hábitat natural.

Un análisis de los principales sistemas geológicos emitidos hasta finales del siglo XVIII fue realizado por Nicolas Desmarest (1725-1815) en el primer tomo de su Encyclopédie Métodique (1794) dedicado a la "Géographie Physique". En este trabajo Desmarest destacaba la importancia que había tenido el diluvio en el diseño de la mayoría de los autores que habían disertado sobre la historia de la Tierra.

De todas formas, el sistema geológico diluvista no fue el único postulado a lo largo del siglo XVIII. En este período varios autores francófonos, como Philippe Bertrand (1730-1811), Louis Bertrand (1731-1812) o Jean-Claude Delamétherie (17431817), discutieron con diferente éxito distintas teorías de la Tierra.

El que realizó este estudio con más detenimiento fue Delamétherie, redactor del Journal de Physique y adjunto a la cátedra de Historia Natural en el Collège de France. Publicó entre otros numerosos trabajos una Théorie de la Terre (Paris, 1795, 3 vols., $2^{a}$ ed. 1797, 5 vols.), en la que tras exponer su teoría de la Tierra - basada en que montañas, valles y llanuras se habían formado por cristalización en las aguas, para lo cual suponía disueltas en ellas todas las materias constituyentes de los continentes -, comentaba en un largo apartado titulado "Des différens systèmes sur la théorie de la Terre" ${ }^{15}$ más de veinte sistemas geológicos propuestos desde los egipcios, hindúes, epicúreos, etc., hasta los de sus contemporáneos.

Su punto de partida era que todos los autores que se habían planteado explicar la formación de la Tierra habían coincidido en afirmar que en un período determinado del pasado todo el globo terráqueo había estado cubierto por las aguas, en cuyo seno se habían formado todas las capas terrestres que componían la actual superficie de la Tierra. Para algunos, el globo había estado en estado incandescente, ya fuera como un sol extinto o como una masa arrancada del sol por el choque de un cometa, etc., pero todos convenían que con posterioridad a este estado de incandescencia la superficie de la Tierra había sido modelada por las aguas ${ }^{56}$.

La mayor parte de las hipótesis partían de que en un principio la superficie terrestre había sido plana, o al menos con accidentes geográficos poco considerables.

55 Delamétherie, J.C. (1795), Théorie de la Terre, Paris, t. III, pp. 340-448.

56 Ibidem, pp. 341 y 441. 
También se suponía en todos estos sistemas geológicos la existencia de cavernas interiores más o menos de gran tamaño. Disentían sin embargo sus autores, a la hora de explicar la formación o el modelado del relieve terrestre, es decir, cómo las aguas que habían sobrepasado las montañas más elevadas habían descendido hasta alcanzar su nivel actual. Las distintas interpretaciones recogidas por Delamétherie cran las siguientes:

1- La superficie terrestre, más o menos plana, había sido surcada por grandes corrientes, que habían excavado los valles.

2- Los valles se habían formado por hundimientos de terrenos.

3- Las montañas se habían levantado debido a fuerzas internas, por lo general volcánicas, y estas elevaciones habían originado los valles.

4- Inmensos maremotos, ocasionados por el levantamiento del suelo submarino, habían depositado los materiales constituyentes de las montañas.

5- Las montañas y los valles se habían formado en el seno de las aguas.

La teoría geológica de Delamétherie fue criticada por el ingenicro de caminos Philippe Bertrand. Con anterioridad Bertrand, que daba gran importancia a la acción geológica del agua, ya había rebatido la teoría de la Tierra de Buffon en su trabajo Lettre a M. le comte Buffon, ou critique et nouvel essai sur la théorie générale de la terre (Besançon y Paris, 1780), y en sus Nouveaux principes de géologie (Paris, 1797, $2^{\mathrm{a}}$ ed. 1804) analizaba críticamente diferentes sistemas geológicos, especialmente el propuesto por Delamétherie.

\section{Los sistemas geológicos neptunista y plutonista ${ }^{57}$.}

En el último cuarto del siglo XVIII surgieron dos teorías de la Tierra basadas en la importancia respectiva que el agua y el fuego - el calor interno terrestre- poseían en el modelado del globo terráqueo. La primera de ellas fue postulada por Abraham Gottlob Werner (1749-1817), profesor de Mineralogía en la Escucla de Minas de Freiberg (Sajonia), para quien todas las formaciones rocosas se habían " constituido en el seno de las aguas por sedimentación de los diferentes tipos. La labor del geólogo era reconocer en todos los lugares las "formaciones universales" o precipitados simultáneos de un disolvente u océano común primitivo, el fluido acuoso. Este sistema, al que se llamó neptunista, tuvo una gran aceptación en su época en

57 Una buena aproximación al debate entre neptunistas y plutonistas puede verse en HALLAM, A. (1985), Grandes controversias geológicas, Barcelona, Labor, pp. 1-28. 


\section{FRANCISCO PELAYO}

Europa continental. Para Werner, las rocas de naturaleza cristalina, como el granito, habían sido las primeras que habían precipitado en el océano primigenio, formándose posteriormente las rocas sedimentarias.

El otro modelo de la dinámica terrestre fue expuesto por James Hutton (1726-1797) en su Theory of the Earth (1788). Según Hutton, tras la erosión, el transporte y el depósito de los materiales sedimentarios en el fondo del mar, el calor interno consolidaba los sedimentos y los elevaba hasta la superficie. Esta sucesión de ciclos geológicos llevaba implícita una idea de eternidad, que se desprendía en la conclusión de su memoria:

"El resultado de nuestra investigación es, pues, que no hallamos ningún vestigio de un comienzo ni perspectiva de un final."

El sistema de Hutton fue conocido como plutonismo debido a la gran importancia que desempeñaba en él la acción del calor interno terrestre. Otra lectura que se deducía de la teoría de Hutton era la idea de eternidad que se desprendía de su uniformitarismo, algo que no fue bien aceptado entre sus contemporáneos por sus implicaciones de una inmensidad del tiempo geológico.

En efecto, la "teoría de la Tierra" que había emitido el escocés Hutton presuponía, por tanto, primero, la existencia de un calor interno que consolidaba los estratos; segundo, la existencia de continuos ciclos geológicos y, en consecuencia, de una uniformidad en la actividad de los agentes físicos y tercero, la necesidad de atribuir a la Tierra una edad indefinida, rozando con la eternidad.

Esta teoría tuvo grandes detractores entre los geólogos denominados por la terminología anglosajona "bíblicos", como el presidente de la Royal Irish Academy, Richard Kirwan (1733-1812), quien practicamente acusó a Hutton de ateísmo. Kirwan, en su On the Primitive State of the Globe and its subsequent Catastrofe (1797), atribuía a la acción modeladora del diluvio el origen y forma de la superficie terrestre.

Otro de los críticos de Hutton, el naturalista ginebrino Jean André De Luc $(1727-1817)^{58}$ basaba sus objeciones en que la eternidad del mundo que se desprendía del postulado no vestige of a beginning, no prospect of an end, manifestaba una posición contraria a la narración de Moisés de la historia de la Tierra. Según De Luc, el análisis de las formaciones geológicas terrestres hablaba en favor de la cronología del Génesis, aparte de proporcionar pruebas de la existencia de una antigua catástrofe que coincidía en el tiempo con el diluvio universal.

58 Ellenberger, F. y Gohau, G. (1981), "A l'aurore de la stratigraphie paléontologique: JeanAndré De Luc, son influence sur Cuvier", Revue de Histoire des Sciences, XXXIV, pp. 217-257. 


\section{La repercusión del debate sobre los sistemas geológicos en España.}

La controversia entre neptunistas y plutonistas sería recogida en España a comienzos del siglo XIX, en algunos artículos publicados en las Variedades de Ciencias, Literatura y Artes. El redactor encargado de los artículos de la sección de Geología de esta revista fue Juan Blasco Negrillo, quien en los ocho volúmenes que salieron de la revista, entre 1803 y 1805 , seleccionó, tradujo y comentó diversos artículos de interés geológico. Uno de estos trabajos discutió sobre la validez de los diferentes sistemas geológicos ${ }^{59}$.

Blasco Negrillo comen $ə a b a$ su artículo manteniendo las grandes dificultades que presentaba diseñar una teoría de la Tierra, que pudiera explicar tanto su formación como la de otros cuerpos planetarios. Porque tal teoría tenía que estar apoyada por hechos o, dicho de otra manera, tenía que ser el resultado de tales hechos, pero como éstos eran tan complicado de relacionar y la mayor parte eran desconocidos, hasta entonces había sido "más fácil imaginar un sistema, que presentar una teoría; y que todas las que se han dado con este nombre han sido hipotéticas, esto es, vagas y como meras probabilidades."60.

Recogía a continuación una división de interpretaciones emitidas sobre el modelado de la superficie terrestre y la formación de las montañas análoga a la realizada por Delamétherie: la de las excavación de los valles por las corrientes de las aguas, la de los hundimientos, la de la formación de montañas y valles por una fuerza interior, la del levantamiento de las montañas por el calor interno y la de la formación de valles y montañas en el seno de las aguas ${ }^{61}$. En este último apartado incluía la teoría de la Tierra postulada por el propio Delamétherie, a la que criticaba que para poder explicar la cristalización de la Tierra, lo que implicaba la disolución de una cantidad enorme de materias, tenía que recurrir a un volumen de agua en el globo terráqueo veinticinco veces mayor que el actual ${ }^{62}$.

El origen de la Tierra, seguía diciendo Blasco Negrillo, también había sido motivo de opiniones muy diferentes: "un antiguo caos y atmósfera de un cometa"; "una masa fluida, compuesta de materias de todas las especies y de todas las figuras posibles"; "una infinidad de átomos que movidos de mil maneras, y después de muchas reuniones diferentes, se juntan al fin y se precipitan los más pesados"; "un sol compuesto del primer elemento puro (esto es el fuego o calórico) que se cubrió de manchas y otros cuerpos oscuros que forman su superficie"; "una parte del sol desprendi-

59 BlasCo NegRiLlo, J. (1803), "Insuficencia de los sistemas geológicos para explicar la Historia de la Tierra", Variedades de Ciencia, Literatura y Artes, t. I, pp. 24-35 y 9I-100.

6i) Ibidem, p. 24

61 Ibidem, pp. 28-35.

62 Ibidem, p. 97. 
da por el choque de un cometa"... Todo esto dejando un lado las opiniones más disparatadas, en las que se había mezclado ciencia y teología ${ }^{63}$.

En sus conclusiones, Blasco Negrillo sostenía que la explicación a tantas interpretaciones se encontraba en que sus autores se habían limitado a dar gran importancia a un solo acontecimiento geológico, a partir del cual habían construido su sistema.

Aunque intentaba manifestarse ecléctico en sus consideraciones, Blasco Negrillo no ocultaba su simpatía por la geognosia werneriana, disciplina cuyo desarrollo permitiría conocer la composición de las masas rocosas de la superficie y corteza terrestres, sus respectiva épocas de formación, la posición y estratificación de las montañas, etc. ${ }^{64}$.

En su opinión, estaba claro, y en esto coincidían todos los geólogos, que la Tierra había estado alguna vez en el pasado cubierta por las aguas, que habían ocupado toda su superficie, "y que desde este diluvio, inundación o gran porción de agua que cubrió todos los continentes, el globo cambió de forma, tanto interna como externa."65

Junto a esta toma de posición neptunista, Blasco consideraba que para conocer la historia de la Tierra era necesario profundizar en el estudio de la mineralogía, recopilar datos, verificar los ya conocidos, rectificar los errores que se descubrieran y apartarse de cualquier sistema, escuela o partido, ya que para él sólo el conjunto global de todos los hechos presentados en la explicación de la configuración histórica de la Tierra, excavaciones naturales, hundimientos, incendio del globo..., podía haber causado las revoluciones que se apreciaban en la superficie terrestre ${ }^{66}$.

\section{CATASTROFISMO, ACTUALISMO Y UNIFORMITARISMO EN EL SIGLO XIX.}

El desarrollo de la Geología durante la primera mitad del siglo XIX estuvo dominado por los debates entre catastrofistas y actualistas y uniformitarios.

El catastrofismo, postulado de una forma coherente por el paleontólogo francés Georges Cuvier (1769-1832), decía en síntesis que del estudio del registro geológico se desprendía que en el transcurso de la historia de la Tierra habían tenido lugar súbitas catástrofes universales, que habían afectado a la superficie terrestre asolando todo a su paso y exterminando a los seres vivos existentes en ese momento. Posteriormente, después de cada catástrofe, la Tierra se había poblado con nuevos organismos, fuera por nuevas creaciones o por emigración desde otras áreas geográficas. La última de estas catástrofes, recogidas en las tradiciones de la mayoría de los pueblos, había sido el diluvio universal descrito en la Biblia.

\footnotetext{
6.3 Ibidem, pp. 97-98.

64 Ibidem, pp. 99-100

6.5 Ibidem, p. 98.

6r Ibidem, pp. 99-100.
} 
Cuvier, en su Discours sur les révolutions de la surface du globe (Paris, 1812), dedicó un apartado a comentar los sistemas emitidos por sus antecesores en el estudio de la historia de la Tierra. En una primera división Cuvier clasificaba a los sistemas geológicos en antiguos y recientes. Entre los primeros incluía a aquellos sistemas descritos hasta mediados del siglo XVIII. Comenzaba diciendo que durante mucho tiempo sólo se habían admitido dos grandes acontecimientos o dos épocas de cambio geológico en el globo terráqueo: la creación y el diluvio. En ese período los esfuerzos de los naturalistas se habían dirigido a explicar el estado actual de la Tierra, partiendo de un determinado estado primitivo que se había visto modificado por el diluvio, imaginando cada uno a su manera la acción, las causas y los efectos de esta inundación. Dentro de este marco geológico limitado por el Génesis, Cuvier consideraba a un primer grupo de autores en los que incluía a los primeros diluvistas: Burnet, Woodward, Whiston y Scheuchzer. Todos ellos habían coincidido en señalar a la inundación universal como el factor determinante en su teoría de la Tierra, aunque cada uno desarrolló su propio sistema ${ }^{67}$.

Cuvier colocaba en un segundo grupo a Descartes y Leibniz, quienes habían considerado que la Tierra había sido un sol extinto, un globo vitrificado, sobre el que al enfriarse los vapores y formarse los mares se habían depositado los terrenos calizos.

La teoría ultraneptunista de Benoit de Maillet, expuesta en su Telliamed (Amsterdam, 1748), según la cual el globo terráqueo durante miles de años había estado totalmente cubierto por las aguas, constituía otro de los sistemas geológicos antiguos considerado por Cuvier, quien recalcaba como Maillet sostenía que los animales terrestres habían sido primeramente marinos, incluso que el hombre había comenzado siendo un pez ${ }^{68}$.

El último de los sistemas antiguos comentado por Cuvier era la teoría de la Tierra de Buffon, de quien decía que se había limitado a desarrollar la propuesta por Leibniz.

En un segundo apartado Cuvier incluía los sistemas modernos, que a su vez subdividía agrupando, por un lado, aquellos geólogos que le parecían haber ido muy lejos en sus propuestas y, por otro, a los que habían postulado sus ideas geológicas en el marco de las ciencias físico-químicas.

Entre los ejemplos de sistemas geológicos más extremos, comenzaba señalando Cuvier que algunos autores habían propuesto sistemas que parecían influidos por las ideas de Maillet, ya que partían de un estado original líquido de la Tierra en el que se

\footnotetext{
67 Cuvier, G. (1985), Discours sur les révolutions de la surface du Globe, Christian Bourgois Éditeur, pp. 61-67; p. 61 .

68 Sobre el Telliamed puede consultarse CAROzZI, A. (1969), "De Maillet's Telliamed (1748): an Ultra-Neptunism Theory of the Earth" in SCHNEER, C.J. (ed.) Toward a History of Geology, Cambridge, pp. 80-99.
} 
había engendrado la vida más simple desde el punto de vista orgánico, tal como las monadas o los infusorios. Posteriormente estos organismos fueron adquiriendo un mayor grado de complejidad orgánica y se diversificaron en diferentes razas, dando lugar a la fauna actual. Cuvier incluía en este grupo los sistemas propuestos por autores alemanes, como T.CH. Rodig, y también la Hydrogéologie (1802) de Lamarck (1744-1829).

Los sistemas geológicos propuestos por otros autores eran organicistas, pues consideraban que el globo terráqueo poseía facultades vitales, en el que las montañas eran sus órganos de la respiración, los esquistos sus órganos de secreción, los filones eran las caries, los minerales productos de la podredumbre, etc. Cuvier mencionaba aquí las ideas fantásticas expuestas por el mineralogista francés E.L.M. Patrin (1742$1815)^{69}$.

Más nuevos aún, decía Cuvier, eran los sistemas panteistas que se podían encontrar en las obras de H. Steffens (1773-1845) o de L. Oken (1779-1851) ${ }^{70}$.

También había divergencia entre los sistemas geológicos propuestos por autores menos imaginativos que los anteriores. En este apartado Cuvier incluía las teorías de la Tierra expuestas por Delamétherie, Hutton, Robert de Paul, caballero de Lamanon $(1752-1787)^{71}$, D. Dolomieu (1750-1801), Marschall ${ }^{72}$ y L. Bertrand ${ }^{73}$.

La crítica que realizaba Cuvier a todos estos sistemas se basaba en que no existía un enfoque global del problema en ninguno de ellos, sino que habían intentado resolver determinadas cuestiones dejando otras pendientes de respuestas.

La teoría de la Tierra de Cuvier tampoco se escapó de la crítica. Le correspondió al geólogo británico Charles Lyell (1797-1875) rechazar las tesis catastrofistas en su obra Principles of Geology (1830-1833). La traducción del título completo de este

69) Ibidem, p. 64

70) Ibidem, p. 65.

71 En su artículo "Description de divers Fossiles trouvés dans les carrières de Montmartre près Paris, \& vues générales sur la formation des Pierres gypseuses", Journal de Physique, t. XIX, 1782, pp. 173-194, Lamanon exponía un sistema geológico que recordaba a la hipótesis de los lagos colgantes de Sulzer. Decía que los continentes habían estado durante mucho tiempo inundados por una multitud de lagos, dispuestos en diferentes niveles, unos encima de otros, que se habían ido desecando sucesivamente, rompiendo sus diques, depositando sus capas conchíferas y desaguando en la cuenca océanica.

72 Cuvier cita el trabajo de Marschall Recherches sur l'origine et le développement de l'ordre actuel du Monde (Giessen, 1802), en el que su autor exponía que piedras estelares, como los meteoritos, al caer en la Tierra constituían las capas de ésta y que manifestaban en los despojos de los seres desconocidos que llevaban la impronta de su origen extraterrestre.

73. El matemático Louis Bertrand fue autor de Renouvellements périodiques des continents terrestres (Hambourg, 1799, $2^{a}$ ed., Géneve, 1803), obra en la que planteaba que la Tierra estaba hueca en su interior y en su centro existía un núcleo imantado que se movía de un polo a otro, arrastrando en este movimiento el centro de gravedad y la masa de los mares, que así inundaban alternativamente los dos hemisferios. 
libro es, poco más o menos, "Principios de Geología, que intentan explicar los cambios ocurridos en el pasado sobre la superficie de la Tierra, por referencias a las causas que actúan en el presente". Esta es la idea básica del actualismo: explicar todos los cambios que habían tenido lugar a lo largo de la historia de la Tierra por la acción de las mismas causas o fenómenos físicos y geológicos que actuaban en el presente.

Además del actualismo geológico, Lyell sostuvo que en el pasado los fenómenos geológicos habían operado con la misma intensidad que lo hacían en el presente, es decir, no habían sido más enérgicos — no se habían dado ni diluvios ni catástrofes universales-, como postulaban los catastrofistas, y habían actuado al mismo ritmo lento y gradual que en la actualidad. De todo esto - causas o fenónemos geológicos del pasado y el presente de la misma naturaleza y actuando con la misma energía y al mismo ritmo lento- se deducía una especie de estado estacionario geológico, una historia de la Tierra uniforme y ahistórica, y de aquí el término de geología uniformitaria con la que se conoce al sistema geológico de Lyell ${ }^{74}$. Asimismo, el ritmo de actuación lento presuponía una gran duración de los tiempos geológicos, que chocaba con los 6.000 años de antiguiedad de la Tierra que se deducía de una lectura literal de la Biblia y que un estricto catastrofismo, por la rapidez de actuación de las causas cataclísmicas, podía asumir facilmente.

En lo que sí coincidieron Cuvier y Lyell fue en hacer una crítica de los anteriores sistemas geológicos. La obra geológica del autor británico comenzaba con una serie de capítulos en los que revisaba los anteriores sistemas geológicos emitidos hasta el siglo XIX.

Ambos sistemas, catastrofismo y actualismo, fueron a su vez revisados y comentados por otros autores contemporáneos, como Thomas Huxley (1825-1895) en $1869^{75}$, e incluso fueron integrados a finales de siglo en una amplia síntesis geológica realizada por Eduard Suess (1831-1914).

Suess, en su Das Antlitz der Erde (Praga, Viena, Leipzig, 3vols., 1883-1909), se interesó en primer lugar por los "movimientos actuales de la corteza exterior del globo", mostrando así que el método actualista estaba bien integrado en las ciencias de la Tierra avanzado ya el siglo XIX, pero tampoco excluyó de su estudio los movimientos bruscos y repentinos y así el primer fenómeno de la Naturaleza por el que se interesa Suess en su obra es el diluvio. Comentó que aparte del relato bíblico se

74 Sobre la valoración de la obra geológica de Ch. Lyell puede consultarse, entre otros trabajos, Gould, S.J. (1987), Time's Arrow, Time's Cycle. Myth and Metaphor in the Discovery of Geological Time, Harvard University Press, Cambridge, Massachussetts. Hay edición castellana: La flecha del tiempo, Madrid, Alianza Editorial, 1992.

75 "Anniversary Address of the President", Quarterly Journal of the Proceedings of the Geological Society, 25, 1869, pp. xxxiii-liii. T.H. Huxley dedicó su discurso presidencial pronunciado en 1869 en la Sociedad Gcológica de Londres a comentar el catastrofismo, el actualismo y el evolucionismo. 


\section{FRANCISCO PELAYO}

conocían textos mesopotámicos relatando un suceso parecido. Suess pensaba que dichas relaciones hacían referencia a un violento ciclón acompañado de un terremoto que habría impulsado el agua del mar al valle Eúfrates. En cualquier caso, para Suess el diluvio no fue un acontecimiento universal. Exponía cómo además de movimientos regulares y actuales también fenómenos excepcionales violentos y catastróficos contribuían a modelar la faz de la Tierra, estableciendo así las bases para diseñar una síntesis entre actualismo y catastrofismo.

\section{EL DEBATE ACTUAL SOBRE SISTEMAS Y MÉTODOS EN LA HISTORIA DE LA GEOLOGÍA}

Durante las últimas décadas, en parte por el interés que despertaron las tesis de Reijer Hooykaas y en parte, sobre todo últimamente, por el nuevo marco catastrofista en el que se interpreta la historia de la Tierra, surgido por las evidencias que sugieren pasados impactos de grandes meteoritos en la superficie terrestre, ha cobrado interés revisar las obras de geología más clásicas con el pretexto de que la historia de esta disciplina científica ha sido escrita recurriendo a una excesiva simplificación, pues históricamente se han asignado papeles de malos a neptunistas y catastrofistas y de buenos a plutonistas y uniformitarios. Los autores que cuestionan este tipo de historia de la geología, algunos desde el campo de la historia de la ciencia y otros desde el de la paleontología, sostienen que las cosas fueron mucho más complejas y hacen hincapié en los métodos empleados por unos y otros para la emisión de sus respectivos sistemas.

En este último sentido, ha sido el historiador de la ciencia holandés R. Hooykaas quien inició esta línea de discusión, planteando la revisión del debate catastrofismoactualismo $^{76}$. La tesis que propone Hooykaas es que debería sustituirse la división entre sistemas o teorías actualistas y catastrofistas por otras cuya base sea la utilización o no de un método actualista en la emisión de cada teoría. De esta forma, se pueden dar dos concepciones en el tratamiento de la historia de la Tierra, una actualista y otra no actualista.

En opinión de Hooykaas, el debate entre catastrofismo y uniformitarismo, a pesar de revelarse como dos sistemas geológicos diferentes, es esencialmente una polémica sobre el método. El catastrofismo mantenía que la interpretación de los datos debía

76 Pueden verse los trabajos HoOYKAAS, R. (1959), Natural Law and Divine Miracle: a historicalcritical Study of the Principle of Uniformity in Geology, Biology and Theology, Leiden, 1959 ( $2^{\mathrm{a}}$ edición, Leiden, 1963; edición francesa Continuité et discontinuité en géologie et biologie, Seuil, 1970) e Ibidem (1970), "Catastrophism in geology, its scientific character in relation to actualism and uniformitarianism", Mededelingen der Koninkluke Nederlandse Akademie van Wetenschappen, Afd Letterkunde Nieuwe Reeks Deel 33, nº 7, pp. 271-316. 
adaptarse a los hechos geológicos, mientras que el uniformitarismo postulado por Lyell tendía a interpretar los datos en conformidad con los supuestos de la inmutabilidad de naturaleza y grado de todas las causas geológicas. De aquí que la afirmación que la oposición entre catastrofismo y uniformitarismo se basa fundamentalmente en la utilización o no de causas naturales, aunque cierta en algunos casos, simplifica a juicio de Hooykaas excesivamente la cuestión, ya que no tiene en cuenta la polémica metodológica ni el hecho de que muchos uniformitarios utilizaron argumentos metafísicos y muchos catastrofistas no lo hicieron en absoluto ${ }^{77}$.

Por su parte, el paleontólogo norteamericano Stephen J. Gould ha hecho hincapié en que el uniformitarianismo es un concepto dual ${ }^{78}$. En un primer sentido, uniformitarianismo sustantivo, es una teoría del cambio geológico que postula la uniformidad del ritmo o condiciones materiales y que surgió contra el sistema catastrofista, introduciendo la noción de cambio lento y acumulativo producido por procesos naturales operantes $^{79}$. En una segunda acepción, uniformitarianismo metodológico, es un principio o procedimiento científico basado en el carácter invariable de las leyes naturales, tanto en el tiempo como en el espacio, que deja a un lado cualquier explicación sobrenatural ${ }^{80}$.

Por último, el también paleontólogo norteamericano G.G. Simpson ha participado en el debate sobre el uniformitarianismo, para lo cual ha intentado definir todas las cuestiones en juego a fin de delimitar una situación tan compleja. En su opinión, las cuestiones que han surgido en las discusiones del uniformitarianismo se recogen en dos clases. A la primera clase pertenecen aquellas cuestiones que tienen que ver con las propiedades inherentes al universo, es decir, con las propiedades inmutables de la materia y de la energía, así como con los procesos y principios que emanan de aqué1las. En resumidas cuentas, con lo que es inmanente en el universo material.

La segunda clase la forman aquellas cuestiones relacionadas con las configuraciones - entendiendo por configuración o contingencia el estado del universo o cualquier parte de él en un momento dado- que han surgido y surgen en la historia.

En la primera clase Simpson incluye al actualismo de Hooykaas y al uniformitarianismo metodológico de Gould, cuestiones que considera equiparables, mientras que a la segunda clase, además del catastrofismo y el evolucionismo, pertenecen el

77 Cf. HoOyKaAs (1970), p. 316

78 GouLd, S. J. (1965), "Is Uniformitarianism Necessary?", American Journal of Science, 263, pp. 223-228.

79 Ibidem, p. 223

80) Ibidem, p. 226. 


\section{FRANCISCO PELAYO}

uniformitarianismo del historiador de la ciencia holandés y el uniformitarianismo sustantivo de su colega y compatriota Gould ${ }^{81}$.

Estas tres posiciones, ejemplos contemporáneos del interés metodológico por la historia de la Geología, no terminan con la cuestión. Muy al contrario, permiten que el debate continúe abierto.

81 SIMPSON, G.G. (1970), "Uniformitarianism. An Inquiry into Principle, Theory and Method in Geohistory and Biohistory", in M.K. Hecht and W.C. Steree (eds.), Essays in Evolution and Genetics in Honor of Theodosius Dobzhansky, New York, pp. 43-96. 